\title{
The impact of non-steroidal anti-inflammatory drugs on the small intestinal epithelium
}

\author{
Osamu Handa, ${ }^{1 * *}$ Yuji Naito, ${ }^{1}$ Akifumi Fukui, ${ }^{1}$ Tatsushi Omatsu ${ }^{1}$ and Toshikazu Yoshikawa ${ }^{2}$ \\ 'Department of Molecular Gastroenterology and Hepatology, Kyoto Prefectural University of Medicine and ${ }^{2}$ Kyoto Prefectural University of Medicine, \\ 465 Kajiicho, Kawaramachi-Hirokoji, Kamigyo-ku, Kyoto 602-8566, Japan
}

(Received 20 September, 2013; Accepted 7 October, 2013; Published online 18 December, 2013)

\begin{abstract}
The small intestine has been called as a dark continent of digestive tract and it had been very difficult to diagnose or treat the disease of small intestine. However recent technological development including video capsule endoscopy or balloon-assisted endoscopy has made us to aware the various diseases of small intestine. By using capsule endoscopy, many researchers reported that more than $70 \%$ of patients treated continuously with nonsteroidal anti-inflammatory drugs (NSAID) exhibit the mucosal damage of small intestine. In some cases, NSAID not only causes mucosal damage but also results in life threatening bleeding from small intestine, which had not been prevented or cured by gastroprotective drug or anti-gastric acid secretion drug administration. Therefore to investigate and identify the effective drug that protects small intestine from mucosal damage is urgently expected. In spite of extensive investigation in clinical field, only a few drugs such as misoprostol, a synthetic prostaglandin $E_{1}$ analogue, has been reported as an effective one but is not satisfactory enough to fulfill the requirement of patients who suffer from NSAID-induced mucosal damage of small intestine. And now, extensive study is being performed using several gastromucoprotective drugs by many researchers. In this review, we introduce the current clinical situation in small intestinal injury of patients under NSAID treatment, and to summarize the molecular mechanism by which NSAID, including acetyl salicylic acid, cause small intestinal damage. In addition, we present results of clinical trials performed so far, and refer the possible preventive method or treatment in the near future.
\end{abstract}

Key Words: small intestine, NSAID, capsule endoscopy, balloon endoscopy, reactive oxygen species

$\mathrm{T}$ he adverse effects of non-steroidal anti-inflammatory drugs (NSAID) on stomach and duodenum have been intensively investigated. However, these effects on small intestine have not been sufficiently investigated. Recent advance in endoscopic technology enabled us to observe small intestinal mucosa more in detail. By using a balloon assisted endoscopy (BAE), such as a double- or a single-balloon endoscopy (DBE or SBE), and a video capsule endoscopy (VCE), we came to know that NSAID cause mucosal damage also in small intestine with relatively high frequency. By using VCE, Graham et al. ${ }^{(1)}$ have found that patients who took various NSAIDs for more than three months exhibit intestinal mucosal damage, compared to $10 \%$ of the individuals who took either acetaminophen alone or nothing as a control group. Maiden et al. ${ }^{(2)}$ also reported by using VCE that $68 \%$ of the 40 healthy volunteers formed intestinal mucosal damage after 14 days medication with diclofenac (a traditional NSAID) and omeprazole (a proton pump inhibitor). In this review, we described the current situation of NSAID-induced intestinal mucosal damage and the molecular mechanism by which NSAID cause intestinal mucosal damage. By clarifying the mechanism together with clinical feature using VCE and BAE, we will be able to find new strategies for preventing NSAID-induced small intestinal damage.

The evaluation method for NSAID-induced intestinal mucosal damage and endoscopic features

NSAID-induced small intestinal mucosal damage shows various endoscopic features such as small shallow round ulcers, punched out ulcers, ring ulcers, irregular shaped ulcers, longitudinal ulcers, membranous stenosis, perforation and so on. ${ }^{(3-5)}$ The location of the NSAID-induced injury in the intestinal lumen is not specific. Multiple lesions are often found.

For the diagnosis of NSAID-induced small intestinal mucosal damage, BAE and VCE is thought to be useful. Matsumoto et al. ${ }^{(6)}$ have reported that BAE is as useful method as VCE in evaluating the severity of NSAID-induced small intestinal injury. Although both methods have its advantages and disadvantages, ${ }^{(7)} \mathrm{VCE}$ can be a relatively stronger tool than BAE for screening the NSAIDinduced small intestinal injury and evaluating the efficacy of candidate drugs on the treatment or prevention of NSAID-induced small intestinal injury, since VCE is less invasive method than BAE. Since we nowadays have some evaluation criteria for the inflammation and damage of small intestinal mucosa, such as that by Graham et al., ${ }^{(1)}$ Goldstein et al., ${ }^{(8)}$ Maiden et al., ${ }^{(9)}$ and Gralnek et $a .^{(10)}$ (Lewis score), we will be able to evaluate directly and precisely the efficacy of candidate muco-protective drugs on NSAID-induced small intestinal injury by using VCE.

On the other hand, BAE can be a stronger tool than VCE for diagnosis and endoscopic treatment, since BAE enable us to examine the lesion as close as possible, to assess histological findings and to perform endoscopic therapy such as balloon dilation for intestinal stenosis or endoscopic hemostasis for intestinal bleeding. In general, NSAID-induced small intestinal ulcer is shallower than that of Crohn's disease. Therefore, the first line therapy for membranous stenosis formed by NSAID-induced ulcer is endoscopic balloon dilation, ${ }^{(5,11)}$ whereas in Crohn's disease, the stenosis is formed by deep ulceration and the endoscopic balloon dilation is not always an adequate therapy. As to endoscopic hemostasis, electrocoagulation, clipping, injection therapy is usually selected based on the endoscopic classification of vascular lesions of the small intesitine. ${ }^{(12)}$ Argon plasma coagulation can burn wide surface but shallow layer of small intestinal mucosa and can be safely used for the lesion such as an angioectasia exhibiting small amount of oozing continuously. On the other hand, clipping is mechanically effective method for the actively bleeding angioectasia. Another therapeutic method is injecting sclerosing

*To whom correspondence should be addressed.

E-mail: handao@koto.kpu-m.ac.jp

He received "SFRR Japan Award of Scientific Excellent" in 2013 in recognition of his outstanding work. 


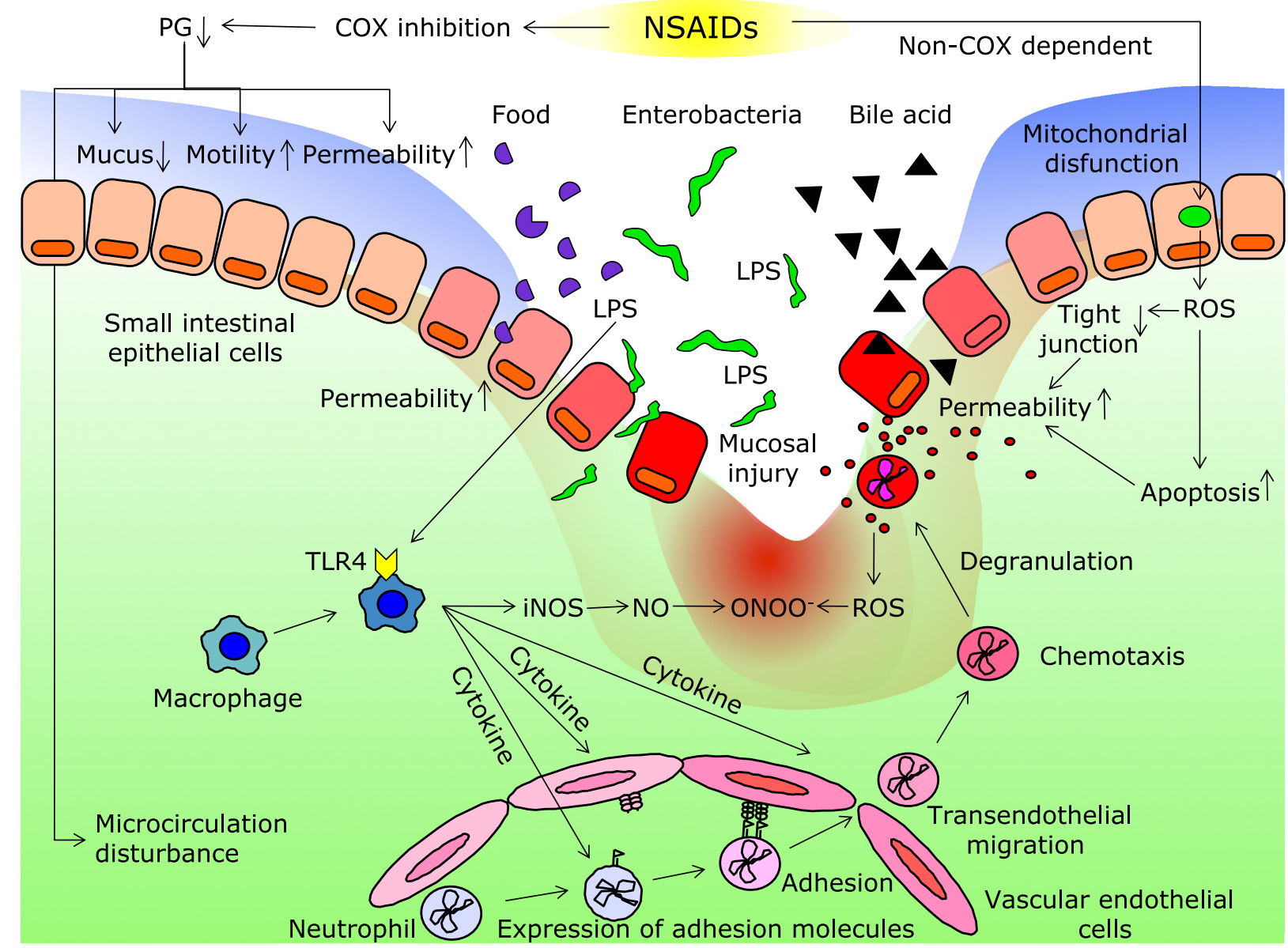

Fig. 1. The figure shows hypothetical molecular mechanisms by which NSAID-induced small intestinal mucosal damage.

agent such as polidocanol (Aethoxysklerol) or ethanolamine oleate around vessels (paravascular injection), ${ }^{(13)}$ however, this indirect hemostasis method is to be examined more in detail.

\section{The molecular mechanism of NSAID-induced intestinal damage}

Although mechanisms by which NSAID induce intestinal damage are multifactorial and therefore difficult to be clearly classified, cyclooxygenase (COX) seems to be greatly involved in the pathogenesis of NSAID-induced intestinal damage. Therefore, in this section, we classified the molecular mechanism in two categories; COX-dependent mechanism and COX-independent mechanism (Fig. 1).

COX-dependent mechanism. NSAID inhibit the intramucosal synthesis of prostaglandins (PG) via inhibiting COX activity at inflamed sites, and thus exhibiting analgesic, antipyretic, and anti-inflammatory effects. In the small intestine, PG regulates gastrointestinal blood flow and mucus secretion, exhibiting mucosal protective functions. Therefore, the inhibition of PG production has been thought to be the major cause of NSAIDinduced small intestinal injury. COX has two subtypes; COX-1 and COX-2. Among these COX subtypes, COX-1 mediated-PG synthesis is especially important for maintaining the mucosal homeostasis. The inhibition of COX-1 has been shown to result in the increment of mucosal permeability, the invasion of intestinal bacteria into mucosa and the synthesis of inducible nitric oxide synthase (iNOS). Inhibition of COX-1 has also been shown to result in the inhibition of intestinal mucosal microcirculation, ${ }^{(14)}$ whereas COX-2 plays a role in the production of PGE2 to protect intestinal mucosa. ${ }^{(15)}$ Therefore COX-1 inhibition is not sufficient for the intestinal mucosal damage. Nowadays, it is widely accepted concept that in addition to the COX-1 inhibition, the COX-2 inhibition is inevitable for the intestinal mucosal damage. ${ }^{(15,16)}$

COX-independent mechanism. As for the COX-independent pathogenic mechanism by which NSAID induce intestinal mucosal damage, Bjarnason et al. ${ }^{(17)}$ proposed "three hit theory". At first, NSAID lyses phospholipids on the mucosal surface and directly injure the mitochondria of intestinal epithelial cells. In the next step, mitochondrial dysfunction results in calcium efflux, free radical formation, reduced intercellular connection, and increased permeability of intestinal mucosa. At the final step, increased intercellular permeability resulted in the intrusion of bile acids, proteases, intestinal bacteria, and toxins that ultimately results in mucosal injury. In addition, Bjarnason et al. ${ }^{(18)}$ concluded that the primary adverse effect of NSAID is to increase the permeability of small intestinal mucosa, and the inflammation or ulcer formation is secondary adverse effect caused by neutrophils that is activated by bile acids or intestinal bacteria.

Recently we have reported that indomethacin (a traditional NSAID) induces reactive oxygen species (ROS) production in mitochondria of small intestinal epithelial cells and apoptosis of these cells that might lead to intestinal mucosal damage ${ }^{(19,20)}$ that is independent of PG suppression. In addition, this ROS production might interact with the other intracellular molecules to cause further injury. Constitutive NOS (cNOS)-derived nitric oxide 
(NO) has been shown to maintain mucosal homeostasis by adjusting mucosal blood flow and mucus secretion. On the other hand, iNOS-derived NO, and ROS may be involved in the ulcer formation of small intestine ${ }^{(21)}$ by interacting each other.

Since NSAID has been reported to undergo entero-hepatic circulation, ${ }^{(22)}$ and Smale et al. ${ }^{(23)}$ emphasized the entero-hepatic circulation of NSAID. They reported that entero-hepatic circulation independent drugs such as sulindac has lower toxicity to small intestinal mucosa. This issue also should be confirmed in further assessment.

Recently many researchers are getting aware of the important role of intestinal bacteria on NSAID-induced small intestinal damage. Accordingly, several groups have reported the importance of the intestinal bacteria as shown in follows. Indomethacin could not cause mucosal damage in germ free rat ${ }^{(24,25)}$ and some antibiotics such as metronidazole ${ }^{(18)}$ and ampicillin $^{(26)}$ or some probiotics (e.g., lactobacillus casei( ${ }^{(27)}$ ) suppressed indomethacininduced intestinal mucosal damage suggesting the pivotal involvement of intestinal bacteria in NSAID-induced mucosal damage. Watanabe et al. ${ }^{(28)}$ reported interesting findings about the active role of toll-like receptor 4 (TLR4) on NSAID-induced small intestinal damage that recognizes lipopolysaccharide (LPS) of gram-negative bacteria and mediates inflammatory activation. They found that indomethacin- and diclofenac-induced intestinal mucosal damage was significantly suppressed in TLR4 mutant mice. This result indicates that NSAID damages intestinal mucosal barriers, causes invasion of gram negative bacteria into mucosa and their LPS activates TLR4 to cause inflammatory reactions such as cytokine production and subsequent chemotaxis of neutrophils. ${ }^{(27)}$

An interesting report has recently been published by Pilotto et al. ${ }^{(29)}$ They suggested that genetic differences might be involved in the increased susceptibility of the small intestinal injury; the polymorphism of CYP2C, an enzyme that metabolizes NSAID, modified the risk of NSAID-related gastroduodenal bleeding. ${ }^{(29)}$ This finding may explain why not all the patients, who are taking NSAID, exhibit the small intestinal damage. This issue should be investigated more in detail with small intestine.

\section{Acetyl salicylic acid induced small intestinal injury}

Among NSAID, acetyl salicylic acid (ASA) has a unique property, which exhibits anti-inflammatory effects at high dose and anti-platelet effects at low dose. Therefore, ASA has been widely used for the secondary prevention of cerebro- and cardio-vascular diseases. However, this useful drug has detrimental effect not only on gastric mucosa but also on small intestinal mucosa. ${ }^{(30)}$

The mechanism by which ASA induces small intestinal mucosa is not clear yet. However the first step of this phenomenon seems to be the increased mucosal permeability. ${ }^{(31,32)}$ Recently, we reported that low concentration of ASA, which does not induce cell death, increases intestinal epithelial cell permeability as assessed by trans-epithelial electric resistance and dextran flux through para-cellular pathway. ${ }^{(33)}$ Moreover, ASA increases the production of reactive oxygen species in the epithelium, which induces the oxidative modification of ZO-1 protein between epithelial cells. By this result, we concluded that ASA could modify the function of tight junction via oxidative stress dependent mechanism. Our preliminary data suggested that drugs that has an anti-oxidant effects might be useful for the prevention of ASA-induced small intestinal epithelial cell permeability (data not shown).

\section{The therapy and the prevention of NSAID-induced small intestinal damage}

The first choice of the treatment for NSAID-induced small intestinal damage is to stop taking NSAID. However NSAID are usually administered to the patients with chronic pain or chronic inflammatory diseases, and stopping these medicines is impossible in many cases.

At the beginning, many people investigated the adverse effect of low-toxic NSAID on small intestine. COX-2 selective NSAID have been reported to cause less damage than non-COX-selective NSAID on small intestine. ${ }^{(8)}$ However, two recent reports have described that there are no differences in the incidence of small intestinal mucosal damage between COX-2 selective NSAID and traditional non-COX-selective NSAID. ${ }^{(9,34)}$

Afterwards, several drugs had been reported to be useful for the therapy and the prevention of NSAID-induced small intestinal injury in animal experiments, and therefore they were expected to be useful in clinical settings, however, only a few drugs has a potential.

Muco-protective prostaglandin analog, misoprostol, that has anti-inflammatory effects, has been also reported to decrease the indomethacin-induced small intestinal mucosal damage via its anti chemotactic effect for neutrophils. ${ }^{(35,36)}$ However these PGE2 drugs have often shows unfavorable side effects such as diarrhea, abdominal pain, or bloating and therefore not suitable for the long use.

The efficacy of sulfasalazine on the NSAID-induced small intestinal damage has been reported, ${ }^{(37)}$ which is a sulfa drug that has anti-inflammatory effects and is commonly used in the treatment of inflammatory bowel disease as well as for rheumatoid arthritis. However, the effect of sulfasalazin were indirectly assessed by blood loss from intestinal wall and therefore should be re-examined by direct observation by VCE or BAE.

As described above, several studies suggested the usefulness of anti-biotics such as metronidazole ${ }^{(18)}$ and ampicillin, ${ }^{(26)}$ however, since in these studies mucosal damage was assessed by measuring mucosal permeability, the clinical usefulness of anti-biotics should be further elucidated. In addition, the usage of anti-biotics may have a limitation: long-term combination therapy with metronidazole and NSAID may lead to the appearance of resistant bacteria, and long time use of ampicillin sometimes causes diarrhea. Therefore, small intestine-specific agents that can be safely used for a long period such as gastric muco-protective drugs are anticipated.

Recent clinical data have shown the usefulness of mucoprotective drugs, rebamipide, on NSAID-induced small intestinal injury. ${ }^{(38)}$ In this prospective, randomized, double-blinded, placebocontrolled and cross-over study, they found that rebamipide administered with omeprazole has protective effect on NSAIDinduced small intestinal injury in healthy volunteer by using VCE. This combination therapy might have a potential and further studies are now performed in our group to confirm the efficacy of this therapy. As described above, our group recently reported that NSAID induces ROS-dependent apoptosis of intestinal epithelial cells ${ }^{(19,20)}$ and ROS-dependent increase of intestinal epithelial cell permeability. ${ }^{(33)} \mathrm{We}$ also found that polaprezinc (PZ), a gastric muco-protective drugs, has a protective effect on NSAIDinduced apoptosis of small intestinal epithelial cells, ${ }^{\left({ }^{39}\right)}$ partly because ROS quenching mechanism of PZ as shown in gastric mucosa. ${ }^{(40-42)}$ Since, in the United States, PZ, a chelating compound consisting of zinc and L-carnosine, is commercially available as a dietary supplement and in Japan PZ is commonly employed as an agent for the treatment of gastric ulcers, we think PZ might be a suitable drug for the treatment of NSAID-induced small intestinal injury.

It is well-known that proton pump inhibitors (PPI) are useful for the treatment of NSAID-induced gastric mucosal damage by its strong anti gastric acid secretion effect, and we have reported that PPI has anti-inflammatory effects, such as the inhibition of interleukin-8 production from vascular endothelial cells and gastric epithelial cells and subsequent chemotaxis of neutrophils. ${ }^{(43)}$ In addition, we and another groups have described the 
protective effect of PPI on NSAID-induced small intestinal injury in the animal experiment. ${ }^{(44,45)}$ Therefore PPI has been expected to be a useful drug for the treatment of NSAID-induced small intestinal injury in the clinical field, however, Goldstein et al. ${ }^{(8)}$ has reported that omeprazole, a PPI, could not prevent NSAIDinduced small intestinal injury in healthy volunteers. Although the effectiveness of PPI might be not high in acid-free environment, another PPI, lansoprazole (LPZ), might have a possibility; Takagi et $a l .{ }^{(46)}$ in our group have reported that LPZ can induce heme oxygenase-1 (HO-1) and this induction might ameliorate NSAIDinduced small intestinal injury. There are no comparative studies in regard to the effect of several PPIs on NSAID-induced small intestinal injury, and clinical trial should be performed to determine whether PPIs are effective or not for the treatment or prevention of NSAID-induced small intestinal injury. In addition, we should determine whether LPZ is suitable for the long use to treat or prevent NSAID-induced small intestinal injury, since LPZ has been reported to cause collagenous colitis in some patients. ${ }^{(47-49)}$ The effect of PPI should also be evaluated because Wallace et al. ${ }^{(50)}$ reported that PPI exacerbate NSAID-induced small intestinal injury by inducing dysbiosis. This issue should be further examined.

\section{Concluding remarks}

In aging societies, the problem of NSAID-induced gastrointestinal damage will become more and more important. And

\section{References}

1 Graham DY, Opekun AR, Willingham FF, Qureshi WA. Visible smallintestinal mucosal injury in chronic NSAID users. Clin Gastroenterol Hepatol 2005; 3: 55-59.

2 Maiden L, Thjodleifsson B, Theodors A, Gonzalez J, Bjarnason I. A quantitative analysis of NSAID-induced small bowel pathology by capsule enteroscopy. Gastroenterology 2005; 128: 1172-1178.

3 Allison MC, Howatson AG, Torrance CJ, Lee FD, Russell RI. Gastrointestinal damage associated with the use of nonsteroidal antiinflammatory drugs. $N$ Engl J Med 1992; 327: 749-754.

4 Kelly ME, McMahon LE, Jaroszewski DE, Yousfi MM, De Petris G, Swain JM. Small-bowel diaphragm disease: seven surgical cases. Arch Surg 2005; 140: $1162-1166$.

5 Kamata Y, Iwamoto M, Nara H, et al. A case of rheumatoid arthritis with protein losing enteropathy induced by multiple diaphragmatic strictures of the small intestine: successful treatment by bougieing under double-balloon enteroscopy. Gut 2006; 55: 1372.

6 Matsumoto T, Kudo T, Esaki M, et al. Prevalence of non-steroidal antiinflammatory drug-induced enteropathy determined by double-balloon endoscopy: a Japanese multicenter study. Scand J Gastroenterol 2008; 43: 490496.

7 Kameda N, Higuchi K, Shiba M, et al. A prospective, single-blind trial comparing wireless capsule endoscopy and double-balloon enteroscopy in patients with obscure gastrointestinal bleeding. $J$ Gastroenterol 2008; 43: 434-440.

8 Goldstein JL, Eisen GM, Lewis B, Gralnek IM, Zlotnick S, Fort JG; Investigators. Video capsule endoscopy to prospectively assess small bowel injury with celecoxib, naproxen plus omeprazole, and placebo. Clin Gastroenterol Hepatol 2005; 3: 133-141.

9 Maiden L, Thjodleifsson B, Seigal A, et al. Long-term effects of nonsteroidal anti-inflammatory drugs and cyclooxygenase- 2 selective agents on the small bowel: a cross-sectional capsule enteroscopy study. Clin Gastroenterol Hepatol 2007; 5: 1040-1045.

10 Gralnek IM, Defranchis R, Seidman E, Leighton JA, Legnani P, Lewis BS. Development of a capsule endoscopy scoring index for small bowel mucosal inflammatory change. Aliment Pharmacol Ther 2008; 27: 146-154.

11 Mehdizadeh S, Lo SK. Treatment of small-bowel diaphragm disease by using double-balloon enteroscopy. Gastrointest Endosc 2006; 64: 1014-1017.

12 Yano T, Yamamoto H, Sunada K, et al. Endoscopic classification of vascular lesions of the small intestine (with videos). Gastrointest Endosc 2008; 67: $169-172$.

13 Tatemichi M, Nagata H, Sekizuka E, Morishita T, Mizuki A, Ishii H. Is
NSAID affects entire gastrointestinal tract, not only small intestine but also stomach and colon, and cause unfavorable symptoms and side effects from epigastric discomfort to mucosal bleeding. Sometimes NSAID cause life threatening side effects without any symptoms in elderly patients. Therefore, agents that can be safely used for the treatment of "pan-gastrointestinal tract" in the patients with NSAID medication for a long period are anticipated.

Since many NSAID users are waiting for the appearance of safety muco-protective drugs, the most important thing for us is to clarify the precise mechanism by which NSAID induce small intestinal damage as soon as possible, and that clarified mechanisms will give us great hints to find novel strategies for preventing NSAID induced small intestinal damage.

\section{Acknowledgments}

This work was supported by Grants-in-Aid for Scientific Research (C) to Y. N. (No. 25460958) from the Japan Society for the Promotion of Science, and by an Adaptable and Seamless Technology Transfer Program through targetdriven R\&D to Y. N. from the Japan Science and Technology Agency.

\section{Conflict of Interest}

Y. N. received scholarship funds from Otsuka Pharmaceutical Co., Ltd., Takeda Pharmaceutical Co., Ltd., and Eisai Co., Ltd. endoscopic paravascular injection of sclerosing agents reasonable in the control of GI bleeding? Gastrointest Endosc 1999; 50: 499-505.

14 Kelly DA, Piasecki C, Anthony A, Dhillon AP, Pounder RE, Wakefield AJ. Focal reduction of villous blood flow in early indomethacin enteropathy: a dynamic vascular study in the rat. Gut $1998 ;$ 42: 366-373.

15 Tanaka A, Hase S, Miyazawa T, Ohno R, Takeuchi K. Role of cyclooxygenase (COX)-1 and COX-2 inhibition in nonsteroidal anti-inflammatory drug-induced intestinal damage in rats: relation to various pathogenic events. J Pharmacol Exp Ther 2002; 303: 1248-1254.

16 Sigthorsson G, Simpson RJ, Walley M, et al. COX-1 and 2, intestinal integrity, and pathogenesis of nonsteroidal anti-inflammatory drug enteropathy in mice. Gastroenterology 2002; 122: 1913-1923.

17 Bjarnason I, Hayllar J, MacPherson AJ, Russell AS. Side effects of nonsteroidal anti-inflammatory drugs on the small and large intestine in humans. Gastroenterology 1993; 104: 1832-1847.

18 Bjarnason I, Hayllar J, Smethurst P, Price A, Gumpel MJ. Metronidazole reduces intestinal inflammation and blood loss in non-steroidal anti-inflammatory drug induced enteropathy. Gut 1992; 33: 1204-1208.

19 Omatsu T, Naito Y, Handa O, et al. Involvement of reactive oxygen species in indomethacin-induced apoptosis of small intestinal epithelial cells. $J$ Gastroenterol 2009; 44: 30-34.

20 Omatsu T, Naito Y, Handa O, et al. Reactive oxygen species-quenching and anti-apoptotic effect of polaprezinc on indomethacin-induced small intestinal epithelial cell injury. J Gastroenterol 2010; 45: 692-702.

21 Bjarnason I, Takeuchi K, Simpson R. NSAIDs: the emperor's new dogma?. Gut 2003; 52: 1376-1378.

22 Bjarnason I, Price AB, Zanelli G, et al. Clinicopathological features of nonsteroidal antiinflammatory drug-induced small intestinal strictures. Gastroenterology 1988; 94: 1070-1074.

23 Smale S, Tibble J, Sigthorsson G, Bjarnason I. Epidemiology and differential diagnosis of NSAID-induced injury to the mucosa of the small intestine. Best Pract Res Clin Gastroenterol 2001; 15: 723-738.

24 Robert A, Asano T. Resistance of germfree rats to indomethacin-induced intestinal lesions. Prostaglandins 1977; 14: 333-341.

25 Whittle BJ, László F, Evans SM, Moncada S. Induction of nitric oxide synthase and microvascular injury in the rat jejunum provoked by indomethacin. Br J Pharmacol 1995; 116: 2286-2290.

26 Konaka A, Kato S, Tanaka A, Kunikata T, Korolkiewicz R, Takeuchi K. Roles of enterobacteria, nitric oxide and neutrophil in pathogenesis of indomethacin-induced small intestinal lesions in rats. Pharmacol Res 1999; 40: $517-524$. 
27 Watanabe T, Nishio H, Tanigawa T, et al. Probiotic Lactobacillus casei strain Shirota prevents indomethacin-induced small intestinal injury: involvement of lactic acid. Am J Physiol Gastrointest Liver Physiol 2009; 297: G506G513.

28 Watanabe T, Higuchi K, Kobata A, et al. Non-steroidal anti-inflammatory drug-induced small intestinal damage is Toll-like receptor 4 dependent. Gut 2008; 57: 181-187.

29 Pilotto A, Seripa D, Franceschi M, et al. Genetic susceptibility to nonsteroidal anti-inflammatory drug-related gastroduodenal bleeding: role of cytochrome P450 2C9 polymorphisms. Gastroenterology 2007; 133: 465-471.

30 Leung WK, Bjarnason I, Wong VW, Sung JJ, Chan FK. Small bowel enteropathy associated with chronic low-dose aspirin therapy. Lancet 2007; 369: 614.

31 Bjarnason I, Williams P, So A, et al. Intestinal permeability and inflammation in rheumatoid arthritis: effects of non-steroidal anti-inflammatory drugs. Lancet 1984; 2: 1171-1174.

32 Smecuol E, Pinto Sanchez MI, Suarez A, et al. Low-dose aspirin affects the small bowel mucosa: results of a pilot study with a multidimensional assessment. Clin Gastroenterol Hepatol 2009; 7: 524-529.

33 Fukui A, Naito Y, Handa O, et al. Acetyl salicylic acid induces damage to intestinal epithelial cells by oxidation-related modifications of ZO-1. Am J Physiol Gastrointest Liver Physiol 2012; 303: G927-G936.

34 Sugimori S, Watanabe T, Tabuchi M, et al. Evaluation of small bowel injury in patients with rheumatoid arthritis by capsule endoscopy: effects of antirheumatoid arthritis drugs. Digestion 2008; 78: 208-213.

35 Bjarnason I, Smethurst P, Fenn CG, Lee CE, Menzies IS, Levi AJ. Misoprostol reduces indomethacin-induced changes in human small intestinal permeability. Dig Dis Sci 1989; 34: 407-411.

36 Hatazawa R, Ohno R, Tanigami M, Tanaka A, Takeuchi K. Roles of endogenous prostaglandins and cyclooxygenase isozymes in healing of indomethacin-induced small intestinal lesions in rats. J Pharmacol Exp Ther 2006; 318: 691-699.

37 Hayllar J, Smith T, Macpherson A, Price AB, Gumpel M, Bjarnason I. Nonsteroidal antiinflammatory drug-induced small intestinal inflammation and blood loss. Effects of sulfasalazine and other disease-modifying antirheumatic drugs. Arthritis Rheum 1994; 37: 1146-1150.

38 Niwa Y, Nakamura M, Ohmiya N, et al. Efficacy of rebamipide for diclofenac-induced small-intestinal mucosal injuries in healthy subjects: a prospective, randomized, double-blinded, placebo-controlled, cross-over study.
J Gastroenterol 2008; 43: 270-276.

39 Omatsu T, Naito Y, Handa O, et al. Inhibitory effect of polaprezinc on indomethacin-induced intestinal epithelial cell injury. Gastroenterology 2008; 134: A529.

40 Yoshikawa T, Naito Y, Tanigawa T, et al. Effect of zinc-carnosine chelate compound (Z-103), a novel antioxidant, on acute gastric mucosal injury induced by ischemia-reperfusion in rats. Free Radic Res Commun 1991; 14: 289-296.

41 Yoshikawa T, Naito Y, Tanigawa T, Yoneta T, Kondo M. The antioxidant properties of a novel zinc-carnosine chelate compound, $N$-(3-aminopropionyl)L-histidinato zinc. Biochim Biophys Acta 1991; 1115: 15-22.

42 Handa O, Yoshida N, Tanaka Y, et al. Inhibitory effect of polaprezinc on the inflammatory response to Helicobacter pylori. Can J Gastroenterol 2002; 16: 785-789.

43 Handa O, Yoshida N, Fujita N, et al. Molecular mechanisms involved in antiinflammatory effects of proton pump inhibitors. Inflamm Res 2006; 55: 476480.

44 Kuroda M, Yoshida N, Ichikawa H, et al. Lansoprazole, a proton pump inhibitor, reduces the severity of indomethacin-induced rat enteritis. Int $\mathrm{J} \mathrm{Mol}$ Med 2006; 17: 89-93.

45 Pozzoli C, Menozzi A, Grandi D, et al. Protective effects of proton pump inhibitors against indomethacin-induced lesions in the rat small intestine. Naunyn Schmiedebergs Arch Pharmacol 2007; 374: 283-291.

46 Takagi T, Naito Y, Okada H, et al. Lansoprazole, a proton pump inhibitor, mediates anti-inflammatory effect in gastric mucosal cells through the induction of heme oxygenase-1 via activation of NF-E2-related factor 2 and oxidation of kelch-like ECH-associating protein 1. J Pharmacol Exp Ther 2009; 331: 255-264.

47 Chiba M, Sugawara T, Tozawa H, et al. Lansoprazole-associated collagenous colitis: diffuse mucosal cloudiness mimicking ulcerative colitis. World $J$ Gastroenterol 2009; 15: 2166-2169.

48 Umeno J, Matsumoto T, Nakamura S, et al. Linear mucosal defect may be characteristic of lansoprazole-associated collagenous colitis. Gastrointest Endosc 2008; 67: 1185-1191.

49 Wilcox GM, Mattia A. Collagenous colitis associated with lansoprazole. $J$ Clin Gastroenterol 2002; 34: 164-166.

50 Wallace JL, Syer S, Denou E, et al. Proton pump inhibitors exacerbate NSAID-induced small intestinal injury by inducing dysbiosis. Gastroenterology 2011; 141: 1314-1322, 1322.e1-e5. 